\title{
X-ray Crystallographic Snapshots of Substrate Binding in the Active Site of Histone Deacetylase 10
}

Corey J. Herbst-Gervasoni and David W. Christianson*

Roy and Diana Vagelos Laboratories, Department of Chemistry, University of Pennsylvania, Philadelphia, Pennsylvania 19104-6323, United States

${ }^{*}$ Correspondence: Email, chris@sas.upenn.edu 
Table S1. Structural summary of HDAC10 complexes

\begin{tabular}{|c|c|c|c|}
\hline Mutation/Substrate & Unmodeled Segments & $\begin{array}{c}\text { B Factor } \\
\text { PDAC }{ }^{\mathrm{a}} \text { Domain } \\
\left(\AA^{2}\right)\end{array}$ & $\begin{array}{c}\text { B Factor } \\
\text { UDAC } \\
\text { Domain }\left(\AA^{2}\right)\end{array}$ \\
\hline Y307F/N ${ }^{8}-A c S p d$ & P369-Q398, G435, A589-K591 & 31 & 51 \\
\hline Y307F/N-AcPut & $\begin{array}{c}\text { P369-D399, G435-D436, T539, } \\
\text { A590-T592, L643 }\end{array}$ & 34 & 53 \\
\hline H137A/N N $^{8}$ AcSpd & $\begin{array}{c}\text { T367-Q398, G435, A589-T592, } \\
\text { L643 }\end{array}$ & 33 & 50 \\
\hline H137A/N-AcPut & P369-Q398, G435, T592-R593 & 31 & 50 \\
\hline Wild-type/Acetate & $\begin{array}{c}\text { P369-Q398, G435, A589-R593, } \\
\text { L643 }\end{array}$ & 33 & 54 \\
\hline
\end{tabular}

aPDAC domain, polyamine deacetylase domain; YDAC domain, pseudo-deacetylase domain. 


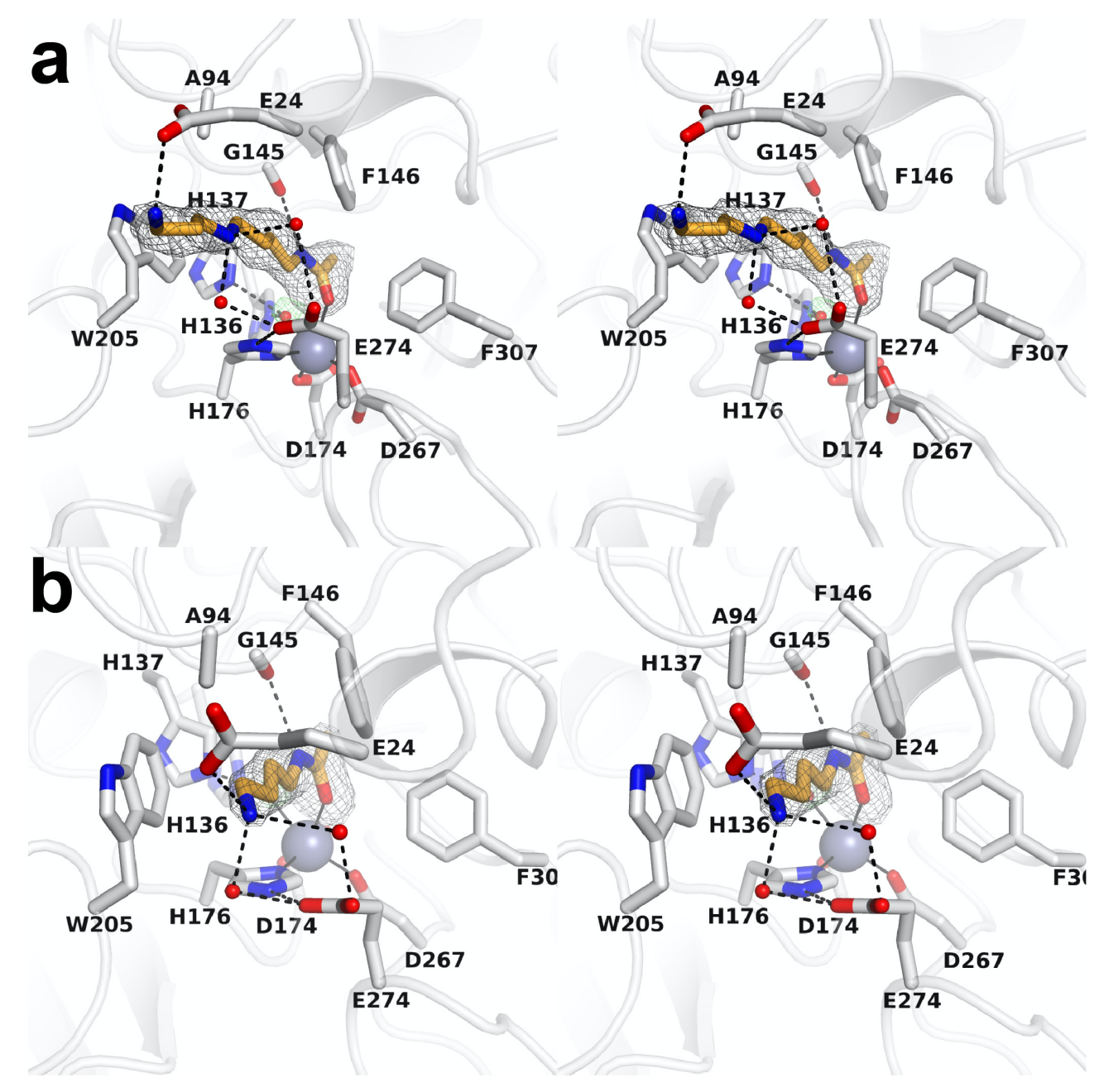

Figure S1. Alternative views of enzyme-substrate complexes shown in Figure 2. (a) Stereoview of Polder omit maps of $\mathrm{N}^{8}$-acetylspermidine (black mesh, contoured at $5.5 \sigma$ ) and the $\mathrm{Zn}^{2+}$-bound water molecule (green mesh, contoured at $8.0 \sigma$ ) in the active site of Y307F HDAC10. Atoms are color-coded as follows: $\mathrm{C}=$ white $(\mathrm{HDAC} 10)$ or orange (substrate), $\mathrm{N}=$ blue, $\mathrm{O}=$ red, $\mathrm{S}=$ yellow, $\mathrm{Zn}^{2+}=$ large gray sphere, water molecule $=$ small red spheres. Hydrogen bonds are shown as black dashed lines and metal coordination interactions are shown as black solid lines. (b) Stereoview of Polder omit maps of Nacetylputrescine (black mesh, contoured at $7.5 \sigma$ ) and the $\mathrm{Zn}^{2+}$-bound water molecule (green mesh, contoured at $9.5 \sigma$ but mostly obscured in this orientation) in the active site of Y307F HDAC10. Atoms are color-coded as in (a). 


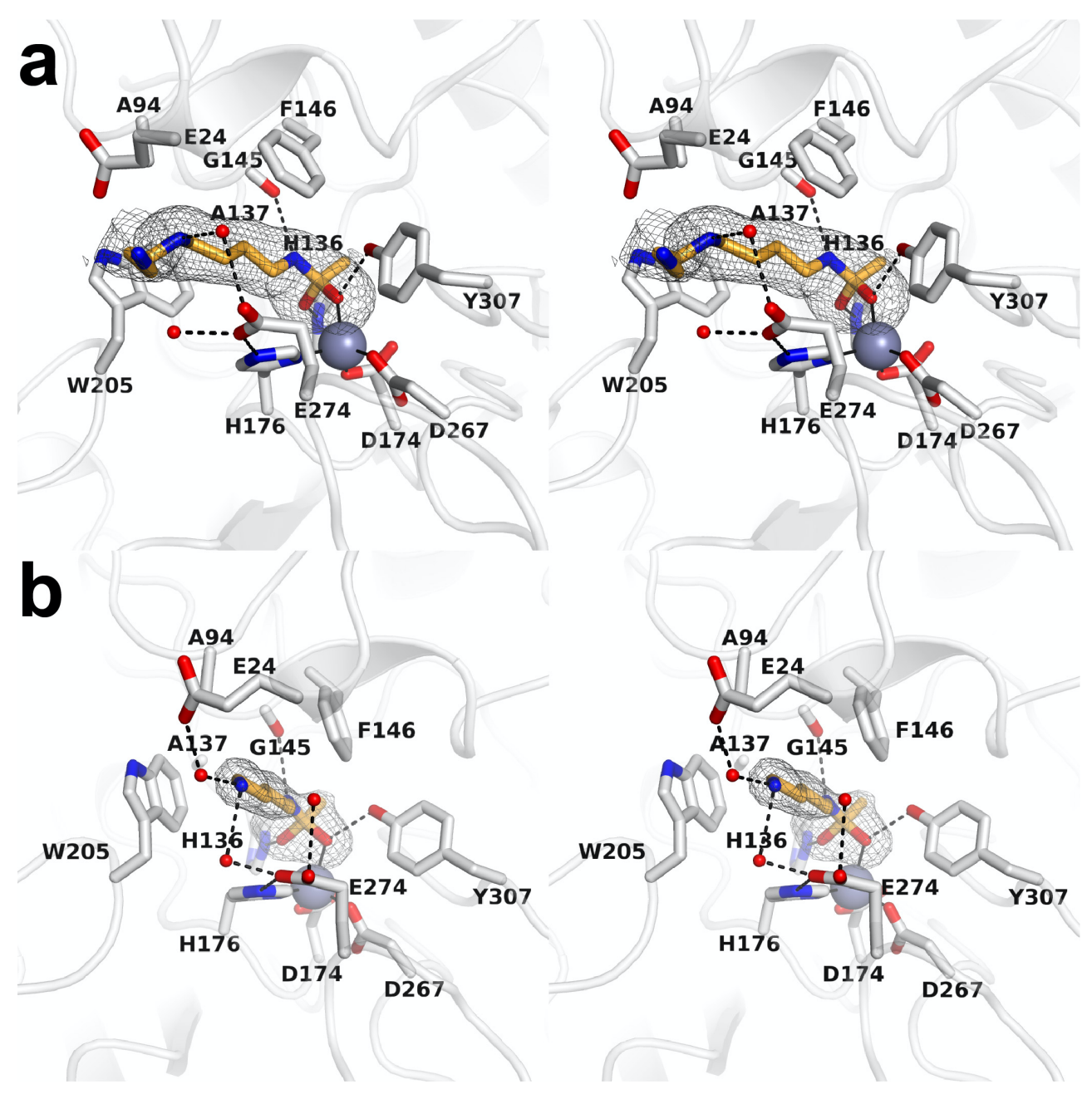

Figure S2. Alternative views of tetrahedral intermediates shown in Figure 3. (a) Stereoview of a Polder omit map of the tetrahedral intermediate of $\mathrm{N}^{8}$-acetylspermidine (black mesh, contoured at $5.5 \sigma$ ) in the active site of H137A HDAC10. Atoms are color-coded as follows: $\mathrm{C}=$ white (HDAC10) or orange (substrate), $\mathrm{N}=$ blue, $\mathrm{O}=$ red, $\mathrm{S}=$ yellow, $\mathrm{Zn}^{2+}=$ large gray sphere, water molecule $=$ small red sphere. Hydrogen bonds are shown as black dashed lines and metal coordination interactions are shown as black solid lines. (b) Stereoview of a Polder omit map of the tetrahedral intermediate of $\mathrm{N}$-acetylputrescine (black mesh, contoured at 7.5 ) in the active site of H137A HDAC10. Atoms are color-coded as in (a). 